\title{
Improvement strategy for robustness of power grid complex network by distributed photovoltaic power system
}

\author{
Xiangying Xie ${ }^{1}$, Dayan $\mathrm{Ma}^{1}$, Zhixiong $\mathrm{Na}^{1}$, Yudong $\mathrm{Lu}^{2}$ and Xin $\mathrm{Luo}^{1, *}$ \\ ${ }^{1}$ State Grid Electronic Commerce Co., LTD. Beijing, 100053, China \\ ${ }^{2}$ State Grid Zhejiang Electric Power Research Institute, Hangzhou 310014, China
}

\begin{abstract}
The robustness of power grids plays a very important role in reality. The blackout of power system can make a serious damage for the normal running of whole city or whole area and the loss caused by it is inestimable. This paper aims to propose a simulation method to evaluate the robustness of a power grid and some effective strategies to improve the robustness. Complex network keeps the most important characters of a power grid, and makes the simulation process concise. This paper studies how characters influence the robustness and proposes some conclusions and suggestions for enhancing effectively the robustness by changing these characters. In this paper, for the robustness of a network, it can be found that some parameters like tolerance coefficient are more dominant than others. Some parameters interact obviously, like capacity addition ratio and addition station ratio. Improper capacity addition strategy may have a negative effect on the network. And load node with big degree and small flow is likely an excellent choice to be added capacity by distributed photovoltaic power station. The simulation method and improvement strategy can be used in the engineering of power grid and establishment of distributed photovoltaic power system.
\end{abstract}

\section{Introduction}

Power grids plays a vital role for development and operation of cities and also people's daily life. Large blackouts of power grids can make huge influences and economic losses directly and indirectly. For example, Fig. 1 shows the August 2003 blackout in Northeastern America disconnected 50 million people and $62 \mathrm{GW}$ to an area spanning eight states and two provinces. The total economic losses of this blackout are estimated at about 25-30 billion dollars. The planning and management of power grids should draw much attention among scientists and engineers.

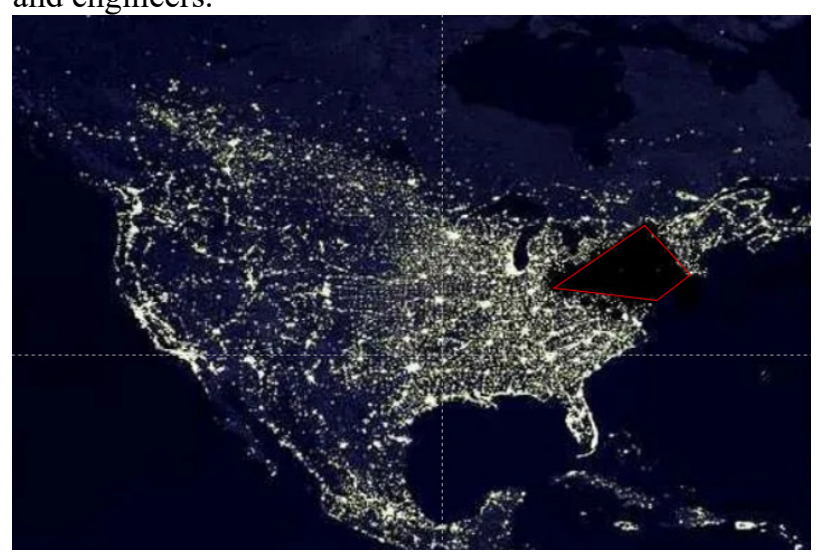

Fig. 1. The great blackout which struck the northeastern United States and Canada on the afternoon of 14 August 2003 and lasted well into the next day.
With the development of complex network theory and its application in recent years, there are many static models of complex network established according to their own characteristics in various fields. For example, traffic flow is introduced into traffic field, and interaction intensity in sociology field. It can be seen that complex network shows remarkable advantages in complex system research.

Electric power network is a network with a large number of nodes and complex connections between them. It has the general characteristics of complex networks, including the large-scale network and the statistical performance of nodes, the complexity of dynamic performance of nodes, the sparseness of network connections, the complexity of connection structure and the complexity of network space-time evolution. As a typical large complex network, with the increasing interconnection scale, the power grid shows more and more complex phenomena, such as frequent large power grid cascading blackouts [1-2] and so on. These accidents are often caused by the failure of one component [3] and then gradually expand, and finally the system collapses rapidly. These system components often appear in overload lines, power flow concentration areas, or loadintensive areas of the system [4]. Distributed photovoltaic power stations are helpful for sharing the load. Distributed photovoltaic power stations usually refer to power generation systems with small installed capacity and located near users using decentralized resources, which have the characteristics of low energy consumption and low cost [5]. This means distributed photovoltaic power

\footnotetext{
* Corresponding author: $\underline{1 \mathrm{x} @ \text { ustc.edu }}$
} 
stations can be set near the important nodes in order to share the load and reduce the risk of cascading failure. For purpose of setting distributed photovoltaic power stations, it's important to find the most important node in the power grid. Therefore, how to evaluate and rank the importance of nodes and how to simulate the cascading failure of the power grid is of great significance to improve the stability and security of the system and reduce the probability of major blackouts.

This paper models the network for power grid and proposes a simulation method to evaluate the robustness of a network based on different problems that the power gird will meet in reality. Then the robustness of a network is discussed by changing the parameters and capacity addition. The importance of each parameter is measured for the robustness of a network and some suggestions and strategies are proposed to enhance the robustness effectively. The study provides some good strategies for setting distributed photovoltaic power stations and a simulation method to measure the improvement of robustness brought by them.

In the next, recent research and related works are introduced in section 2; evaluation and simulation methods are proposed in section 3; simulation results and discussion in section 4; and a conclusion in section 5 .

\section{Recent research}

Recently a great deal of attention has been devoted to the analysis of the importance of nodes in the power grid. Many classical node importance assessment models have been used in power grid analysis, such as PageRank algorithm [6], node importance evaluation matrix [7], degree centrality, betweenness centrality, closeness centrality, eigenvector centrality [8-11] and so on. Researchers basically modeled based on the structure of the network [12]. However, the classical models cannot accurately represent the electrical characteristics of the power grid. To take such characteristics into consideration, many improved models have been developed [13-14]. For instance, the method based on power tracing and link analysis has been followed by researchers [15-17]. Based on the results of power flow tracing, the research defined new indexes for evaluating the link strength and load importance of system nodes. Referring to PageRank algorithm based on link analysis, researchers presented the evaluation model of node importance based on weighted digraph link strength of power grid [15,18]. A method for identifying key nodes of power grid based on improved node importance contribution matrix is also proposed [19]. In June 2019, an evaluation method based on interdependent network theory is presented [20]. The single side network is extracted from the power physicsinformation network, and the subnet models are established with the link-used rate and power line impedance value as the edge weight parameter. Research [21] verifies the feasibility of node shrinkage method for evaluating the importance of network nodes.

Besides the node importance evaluation model, the cascade failure simulation also plays an important role in the power grid analysis [22]. In 1987, Bak, Tang and
Wiesenfeld presented the sand pile model by studying self-organizing critical state [23]. K-core model has also been employed for understanding cascade failure and systems collapse [24]. In order to describe the transition of power grid from initial state to self-organized critical state, Dobson, et al. proposed OPA model [25]. This model is used to model and analyze the changes that may occur during the evolution of power grid state, such as the increase of user load, the change of power grid capacity, the repair of faults and the control of power distribution when faults occur. According to the change of probability distribution of successive fault frequency and fault scale in the process of load increase of power grid, Dobson, et al. presented cascade model [26]. CML model is also widely used to simulate cascade failure [27-28]. In order to improve $\mathrm{K}$-core model, $\mathrm{KQ}$ model is proposed and applied in the research. This cascading failure model is an extension of the k-core cascade, where a node will be removed from the network with a probability $f$ if it has fewer than ks connections, or it has lost more than a fraction $1-\mathrm{q}$ of its original neighbors [29-30].

\section{Evaluation method of the robustness of a network}

\subsection{Architecture of power grid as complex network}

For making simulation results pellucid, we simplify the model of the power grid as a complex network of highvoltage transmission lines which connect power stations, excluding low-voltage transmission lines and some power station's details. A node can be generator, load or pivot. Fig. 2 shows a six nodes power grid network for example.

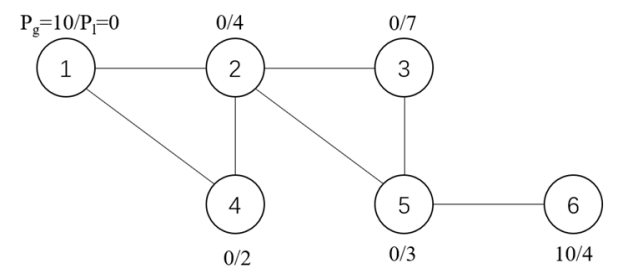

Fig. 2. A six nodes power grid network, it shows node number, connection relation, generated power and load power of nodes in the power grid.

After simplifying the model, a method is proposed to infer the transmission relation of network by linear programming. Considering the influence of the loss for transmission relation and supposing the loss ratio on each edge is similar, a generator should have a priority to provide energy to nearer load. We define distance between node $\mathrm{i}$ and $\mathrm{j}-\mathrm{D}_{\mathrm{ij}}$ as the number of edges in the shortest path from $i$ to $j$, transmission power from generator $i$ to load $\mathrm{j}--\mathrm{X}_{\mathrm{ij}}$ and transmission power between two neighbor nodes $\mathrm{i}$ and $\mathrm{j}-\mathrm{P}_{\mathrm{ij}}$ ( $\mathrm{i}$ and $\mathrm{j}$ are connected). The objective and constraints of the linear programming problem are shown in equation (1).

$$
\max S=\sum_{i \in G} \sum_{j \in L} \frac{X_{i j}}{D_{i j}}
$$




$$
\text { s.t. }\left\{\begin{array}{c}
\left(\sum_{j \in L} X_{i j}\right) \leq P_{i}^{g}, i \in G \\
\left(\sum_{i \in G} X_{i j}\right) \leq P_{j}^{l}, j \in L
\end{array}\right.
$$

\subsection{Node's characters}

In the study, two characters of nodes in network are significant -- degree $\mathrm{k}$ and flow $\mathrm{S}$. The definition of degree is the same as that in graph theory. Generated power $\mathrm{P}_{\mathrm{g}}$, load power $\mathrm{P}_{1}$, provide value and taken value are also useful for the experience and definitions of other important parameters.

(1)Flow $\mathrm{S}$

The flow $\mathrm{Sj}$ is a character of node $\mathrm{j}$ for measuring the maximum power output capacity, which represents the sum of generated power of node $\mathrm{j}$ and all power flow in node $\mathrm{j}$.

$$
S_{j}=P_{j}^{g}+\sum_{i \in N} P_{i j}
$$

(2) Generated power Pg and provide value

Generated power $\mathrm{P}_{\mathrm{g}}$ is the maximum power generation capacity of a normal working node. Provide value means the value of power that a generator node provides exactly to other load nodes or itself. In the next part, 'provide' is short for provide value.

(3) Load power Pl and taken value

Load power $\mathrm{P}_{1}$ is the maximum load capacity of a normal working node. Taken value means the value of power that a load node taken exactly from other generator nodes or itself. In the next part, 'taken' is short for taken value.

\subsection{Network's robustness evaluation}

Robustness refers to the ability of tolerating perturbations that might affect the system's functional body. There are two types of perturbations in a power grid. The first one is one station lose all its functions. The second type is cascading failure. Therefore, in this section two method of evaluating network's robustness are proposed: node breakdown and cascading failure.

(1) Node breakdown

Node breakdown includes two types of attack mode - removing the node and all edges link with it; stopping its function of generation and load but remaining transmits function, which is shown in Fig.3.

$\mathrm{C}_{\mathrm{C}}$ is the ratio of actual total power of a network against maximum total power of the original network before the attack. Bigger $\mathrm{C}_{\mathrm{C}}$ shows better connectivity of a network. $C_{L}$ is the average distance of electric power transmitted from generators to loads. And $\mathrm{C}_{\mathrm{g}}$ is a summary measure of $\mathrm{C}_{\mathrm{C}}$ and $\mathrm{C}_{\mathrm{L}}$.

$$
\left\{\begin{array}{c}
X_{N}=\sum_{i \in G} \sum_{j \in L} X_{i j} \\
C_{L}=\sum_{i \in G} \sum_{j \in L} \frac{X_{i j}}{X_{N}} \times D_{i j} \\
C_{g}=C_{C} / C_{L}
\end{array}\right.
$$

In conclusion, choose one attack mode to attack a node each time and get $\mathrm{C}_{\mathrm{C}}$ and $\mathrm{C}_{\mathrm{g}}$ two metrics before and after the attack. Then calculate the decline of two metrics during the attack. Bigger decline shows bigger destructiveness of the attack and weaker robustness of the network.

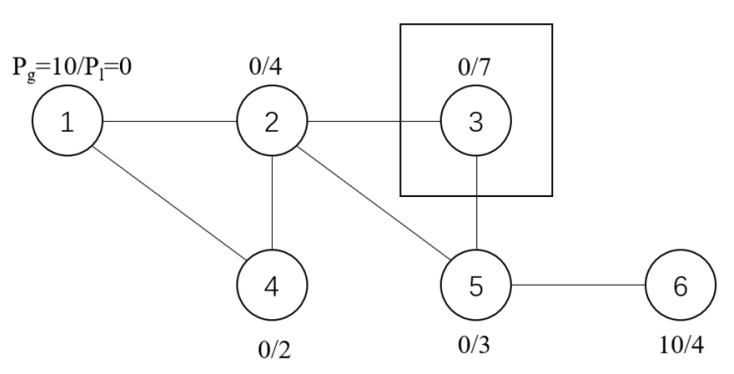

(a)

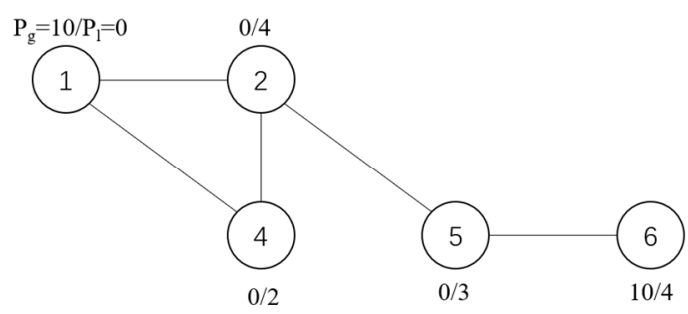

(b)

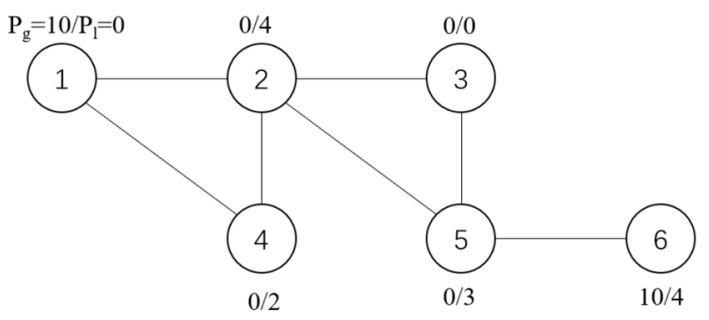

(c)

Fig. 3. Schematic of node breakdown. (a) Node breakdown of node 3. (b) removing the node and all edges link with it. (c) remaining transmits function.

(2) Cascading failure

Supposing the capacity of load is positively related with load power $\mathrm{P}_{1}$ for a node, $\alpha$ is tolerance coefficient of the node which is more than zero.

Select one node that has not been attacked each time to augment its load power. According to the node status and allocation strategy given by equation (5), the power of the node is allocated. Re judge the distribution of all nodes with increased load until the load power of all nodes in the network no longer changes, record the number of overload nodes, fault nodes and $S_{\mathrm{r}}$ given by equation (6) which is the sum of the work efficiency of each node and shows network's completeness.

$$
\left\{\begin{array}{c}
\Delta l_{j k}=\left(l_{j}-\varepsilon c_{j}\right) \tau_{j k} \\
\tau_{j k}=\frac{c_{k}-l_{k}}{\sum h \in N_{\text {normal }} c_{h}-l_{h}}
\end{array}\right.
$$

$\varepsilon$ is rest coefficient which represents a node's robustness in overload state. When a node is in failure state, $\varepsilon$ is zero. Nnormal is set of all neighbor nodes of node $\mathrm{j}$ which are in normal working state

$$
\begin{gathered}
S_{r}=\left(\sum_{i \in N} s_{i}\right) / \operatorname{size}(N) \\
s_{i}=\left\{\begin{array}{c}
0, \text { failure state } \\
\frac{\delta c_{i}-l_{i}}{\delta c_{i}-c_{i}}, \text { overload but not in failure state } \\
1, \text { normal state }
\end{array}\right.
\end{gathered}
$$

Overload coefficient $\delta$ represents the capacity of processing extra load beyond c for nodes in the network. In other words, $\delta c_{i}$ is maximum possible load value for node $i$. If $l_{i}>\delta c_{i}$, node $i$ must be in failure state, if 
$c_{i}<l_{i}<\delta c_{i}$, node $\mathrm{i}$ is in overload state and has a probability to be in failure state, the probability is called failure probability in the paper and it is constant for all nodes of a network.

Finally, for each node in the list of nodes to be attacked, a set of nodes will be recorded. Fig.4 shows how these results measure the network's robustness when the network faces to cascading failure.

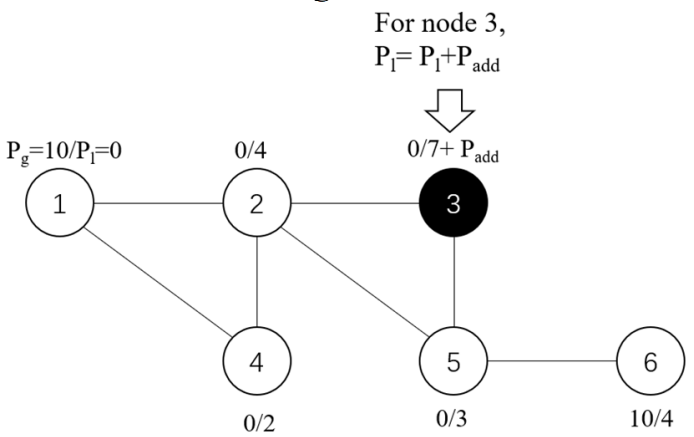

(a)

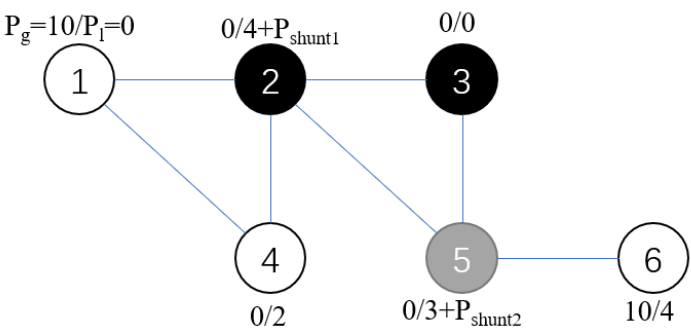

(b)

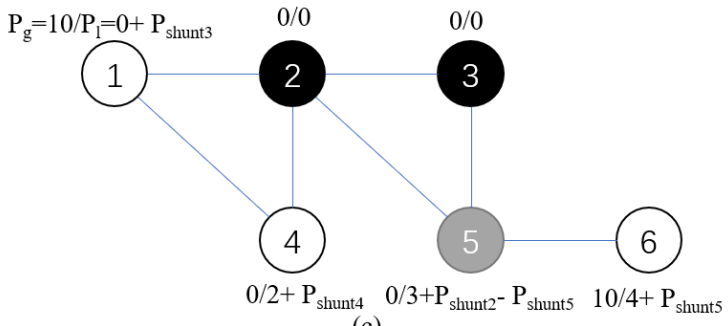

(c)

$$
\begin{aligned}
& \mathrm{P}_{\text {shunt } 1}+\mathrm{P}_{\text {shunt } 2}=7+\mathrm{P}_{\text {add }} \\
& \mathrm{P}_{\text {shunt } 3}+\mathrm{P}_{\text {shunt } 4}=\mathrm{P}_{\text {shunt } 1}+4
\end{aligned}
$$

(d)

Fig. 4. Schematic of cascading failure. (a) Load power of node 3 augments suddenly and the network's balance is broken. (b)Node 3 is into failure, its load power is shunted to neighbor nodes, so load power of node 2 and 5 augment suddenly. Node 2 is in failure state and node 5 is in overload state. (c) Cascading failure influences other nodes in the network, some nodes are in overload state (like node 5), some nodes are in failure state (like node 2 and 3 ) and others are in normal working state (like node 1, 4 and 6). (d)The relation of shunt of load power between neighbor nodes.

\subsection{Simulation process}

Using evaluation methods mentioned in section 3.3, we can study the robustness of a network to the addition of capacity and the network's parameters.

(1) Simulation of attacks.
For the simulation of attacks, two types of evaluation methods are mentioned: node breakdown and cascading failure. Different attack strategies are used to attack the network specified times, then taking the average of these evaluation results as the final result. Three attack strategies are proposed. In random strategy, every node has a same probability to be chosen. In preference of flow $\mathrm{S}$ strategy and preference of degree $\mathrm{k}$ strategy, the probability of being chosen is positively correlated with flow $\mathrm{S}$ and degree $\mathrm{k}$ respectively.

(2) Capacity addition.

Establishment of local and supplementary power station is widely used to enhance a network's robustness. An example of the capacity addition of a network is shown in Fig.5.

The addition strategies are composed by three decisions $(2 \times 2 \times 4=16)$. Metrics includes the node's flow $\mathrm{S}$ and degree $\mathrm{k}$. Order direction decides whether to choose the biggest value node first or the smallest value node first. Preference decides that which kind of nodes is preferred (generator, load or pivot) to be added capacity or be treated equally.

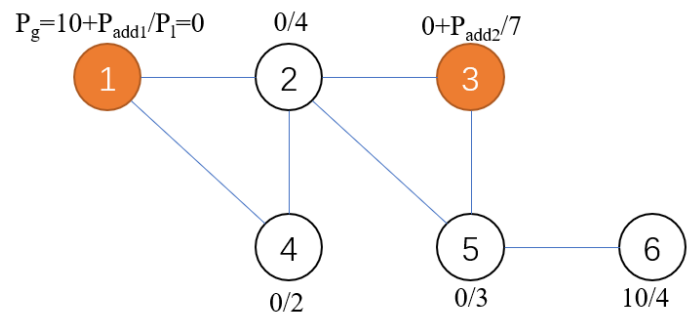

Fig 5. Capacity addition. Choose some nodes by addition strategies and make their generated power is augmented. In reality, establishment of local and supplementary power stations like distributed photovoltaic power stations can realize capacity addition.

\section{Results and discussion}

\subsection{Default setting of simulation}

There are many of optional parameters for the simulation, so default setting of these parameters should be chosen at first. The default setting of these parameters mainly bases on two standards. The first standard is that default parameter should be close to real situation. The second one is that default parameters should be good for making results of simulation distinct. In this paper, tolerance is 0.6 , overload coefficient is 1.5 , failure probability is 0.5 , rest coefficient is 1 and overload ratio is 1 as default setting.

Reverse or not is a parameter used in our different strategies. When reverse is false, nodes are sorted from big metrics value to small metrics value. When reverse is true, nodes are sorted from small metrics value to big metrics value.

\subsection{Parameters and results in simulation of cascading failure}


In simulation of cascading failure, failure node number, overload node number and $\mathrm{Sr}$ are recorded.

(1) Tolerance coefficient

Table 1. Simulation result with different tolerance coefficient

\begin{tabular}{cccc}
\hline tolerance & \multicolumn{3}{c}{ result } \\
\cline { 2 - 4 } & failure & overload & $\mathrm{S}_{\mathrm{r}}$ \\
\hline 0.3 & 3.3089 & 3.8623 & 0.8853 \\
0.4 & 3.0591 & 3.467 & 0.8937 \\
0.5 & 2.5923 & 3.007 & 0.9104 \\
0.6 & 2.2753 & 2.7373 & 0.921 \\
0.7 & 2.0855 & 2.4176 & 0.9266 \\
0.8 & 1.914 & 2.2891 & 0.9324 \\
\hline
\end{tabular}

From the simulation result, it can be observed that, firstly, tolerance coefficient is a significant parameter for the robustness of a network. Bigger tolerance coefficient brings a stronger robustness when the network faces to danger of cascading failure. Secondly, 0.6 seems like a threshold value of tolerance coefficient for this model. Therefore, for a network whose tolerance coefficient is less than 0.6, augmentation of tolerance coefficient is likely a good choice to enhance the network's robustness.

(2) Overload coefficient and failure probability

Exactly as introduction in 3.3, overload coefficient and failure probability are in relation of mutual cooperation. That's why the two parameters are discussed together.

From the simulation result, it can be observed that, firstly, bigger overload coefficient and smaller failure probability bring a stronger robustness. Secondly, if any parameter in the two is bad, it will influence the network's robustness badly though the other parameter is very good. Thirdly, failure probability plays a more important role in the robustness of a network than overload coefficient. As a conclusion, if we want to enhance the robustness of a network by ameliorating overload coefficient and failure probability, ameliorating of worse one is the first choice. If there isn't bad parameter or they can't be judged easily, ameliorating of failure probability can get a better result likely.

(3) Rest coefficient

From the simulation result, it can be observed as follow. Firstly, there is no doubt that bigger rest coefficient brings a stronger robustness. Secondly, rest coefficient isn't as significant as other parameters for the robustness of a network. The augmentation of $S_{r}$ is only 0.0019 when rest coefficient increases from 0.6 to 1 . As a reference, the augmentation of $\mathrm{S}_{\mathrm{r}}$ is 0.0106 when tolerance coefficient increases from 0.5 to 0.6 .

(4) Overload ratio

Unlike other parameters, overload ratio hasn't influence for the robustness of a network, because it is just a parameter of the simulation of attack rather than a parameter of the network. But from the results, we can observe that, firstly, when overload ratio is smaller than 0.5 , the danger and damage of cascading failure are slight. When overload ratio is around 1, the danger and damage are acceptable. But when overload ratio is close to 1.25 even exceed to 1.25 , the danger and damage are considerable. In reality, when considering about cascading failure problem of power grid, we can ignore suitably perturbations whose overload ratio is less than 0.5 , and avoid perturbations whose overload ratio is more than 1.25 as far as possible.

\subsection{Results of capacity addition}

In this section, computer simulations are conducted with different addition strategy and addition parameters. We discover and discuss the difference of strategies and how these parameters influence the result of simulation.

(1) Addition strategy

From the simulation result, some interesting phenomena can be observed. Firstly, results from simulation of node breakdown and simulation of cascading failure have consistency basically. The difference of results from simulation of cascading failure is more distinct and representative.

Secondly, for simulation of node breakdown, strategies whose reverse is true have a better effect for enhancing the robustness of a network. It's reasonable, because more important node is more likely to meet trouble no matter in our simulations or in reality. And node breakdown simulate that a node loses its function directly, so make an important node more important isn't an effective way to enhance the robustness, sometimes it is even counter-productive. On contrary, adding capacity for less important nodes and make the network more balanced can get a better effect.

Thirdly, three types of node are good choice to add capacity for enhancing the robustness of a network: nodes that have a big degree, nodes that have a small S and aren't pivot node, pivot nodes that have a big $\mathrm{S}$.

Fourthly, preference doesn't play an important role in these results, except when we set $\mathrm{S}$ as metrics and preference as pivot, there is an obvious otherness. No matter what we set preference from none, generator and load, there isn't a big distinction. But if we must analyze which preference is better from these results, setting load as preference seems like a good choice.

(2) Addition ratio and addition station ratio

From the simulation result, it can be observed that, firstly, the two parameters interplay, and change of one parameter can't decide directly how the robustness of a network change. When addition station ratio is small, the upper limit that the robustness can be promoted is relatively low. When addition ratio is small, the exact augmentation of robustness brought by capacity addition is finite, no matter we concentrate them in a spot of stations or disperse them to a lot of stations.

Secondly, when addition ratio and addition station ratio increase at same time, the result has a distinct improvement.

Thirdly, bad choice of addition node can lead to a negative effect, especially when addition ratio is small, because the load node with weak robustness is weakness of a network in cascading failure and a bad capacity addition process can generate more such weakness. To avoid this condition, setting load node as preference is a good choice. 
Table 2. Simulation result with different addition ratio and addition station ratio (addition strategy: metrics is $\mathrm{S}$, preference is none and reverse is true)

\begin{tabular}{llllllll}
\hline \multirow{2}{*}{ addition ratio } & \multirow{2}{*}{ station ratio } & \multicolumn{3}{c}{ node breakdown } & \multicolumn{3}{c}{ cascading failure } \\
\cline { 3 - 8 } & & $\mathrm{C}_{\mathrm{C}}$ & $\mathrm{C}_{\mathrm{g} \_}$ori & $\mathrm{C}_{\mathrm{g}}$ & failure & overload & $\mathrm{Sr}$ \\
\hline Before capacity addition & 0.9106 & 0.3286 & 0.2964 & 2.3486 & 2.7734 & 0.9188 \\
0.05 & 0.05 & 0.9148 & 0.3394 & 0.3091 & 2.2449 & 2.7061 & 0.9224 \\
0.05 & 0.15 & 0.9093 & 0.3394 & 0.3101 & 2.2731 & 2.7251 & 0.9217 \\
0.05 & 0.25 & 0.9081 & 0.3394 & 0.3087 & 2.6258 & 3.0653 & 0.91 \\
0.15 & 0.05 & 0.9226 & 0.3601 & 0.3312 & 2.2004 & 2.6002 & 0.9239 \\
0.15 & 0.15 & 0.9161 & 0.3601 & 0.3319 & 1.7506 & 2.2771 & 0.9388 \\
0.15 & 0.25 & 0.9202 & 0.3601 & 0.3337 & 2.1175 & 2.8165 & 0.9265 \\
0.25 & 0.05 & 0.9203 & 0.3796 & 0.3501 & 2.2295 & 2.6283 & 0.9232 \\
0.25 & 0.15 & 0.9214 & 0.3796 & 0.3537 & 1.3176 & 1.6905 & 0.9538 \\
0.25 & 0.25 & 0.9239 & 0.3796 & 0.3539 & 1.5086 & 2.1226 & 0.9473 \\
\hline
\end{tabular}

\section{Conclusion}

In this paper, a model method for power grid is proposed to evaluate the robustness of a network. Then how to enhance the robustness of a network is discussed. For example, changing the parameters setting or adding capacity.

The simulation method has two advantages. First, the simulation result shows not only the change of parameters in damaged node, but also the interaction between nodes and the change of whole network. Second, the simulation method refers to two different evaluation methods, it can get a more all-sided result. We measure the importance of each parameter for the robustness of a network and propose some suggestions and strategies to enhance the robustness effectively.

It can be found that capacity addition with good strategy and value setting is an excellent way to enhance the robustness of a network. Distributed photovoltaic power station is very appropriate to realize the goal. Our study provides some good strategies for setting distributed photovoltaic power stations and a simulation method to measure the improvement of robustness brought by them. The simulation results in this article base on IEEE 30, and same conclusions appear with IEEE 57 and IEEE 118. The general tendency of simulation results for three different power grids is similar, which means the conclusions have generality. This paper also uses other simulation methods mentioned by other papers to simulate with same power grid network and the simulation results and conclusions are more or less similar. The simulation method and improvement strategy can be used in the engineering of power grid and establishment of distributed photovoltaic power system.

In the future, in order to get more comprehensive conclusion, the study of power grid complex network can consider more characters like network's mean degree and import more physical relations and parameters of power grid.

\section{Foundation Items}

National Key R\&D Program of China (No.2018YFB1500800); Science and Technology Projects from State Grid Corporation: Deepening Research and Application of State Grid Distributed Photovoltaic Cloud Network (52110418001C).

\section{References}

1. Shi Libao, Shi Zhongying, Yao Liangzhong, Ni Yixin, Masoud Bazargan. Power System Technology, 34, 3 (2010)

2. Vespignani A. Nature, 464, 7291 (2010)

3. Mingtian Du, Hongmei Gao, Lili Li, Bo Wang, Huaqiang Li, Meijun Liu IOP Conference Series: Earth and Environmental Science 227, 3 (2019)

4. Shi Jin Research on Power System Network Model and Network Performance Analysis Based on Complex Network Theory Hubei: Huazhong University of Science and Technology (2008)

5. Zhang, J., Wei, X. IOP Conference Series: Earth and Environmental Science 227, 2 (2019)

6. Sehgal U, Kaur K, Kumar P. International Conference on Computer \& Electrical Engineering. IEEE (2009).

7. Zhou Xuan, Zhang Fengming, Li Kewu, Hui Xiaobin, Wu Husheng Acta Physica Sinica, 61, 5 (2012)

8. Wang Bing. The research of important nodes measuring algorithm for complicated networks Nanjing: Nanjing University of Posts and Telecommunications (2015)

9. Liu Dichen, Ji Xingpei, Wang Bo, Tang Fei Topological vulnerability analysis and countermeasures of electrical communication network based on complex network theory Power System Technology, 39, 12 (2015)

10. Liu Dichen, Ji Xingpei, Chen Guo, Wang Bo, Wei Daqian Link addition strategy based on complex network theory for power communication network Electric Power Automation Equipment, 36, 10 (2016) 
11. Zhang Zhidong, Yang Ting. Power communication backbone network critical elements recognition algorithm based on complex network theory Electric Power Information and Communication Technology, 13, 12 (2015)

12. Shan Zhengbo, Wang Huifang, Lin Guanqiang, He Benteng Identification of vulnerable lines in power grid considering relative probability and consequence of line outage Journal of Zhejiang University (Engineering Science), 52, 3 (2018)

13. Kim J, Bucklew J A, Dobson I. Splitting method for speedy simulation of cascading blackouts IEEE Transactions on Power Systems, 28, 3 (2013)

14. Chen Q, Mili L. Composite power system vulnerability evaluation to cascading failures using importance sampling and antithetic variates IEEE Transactions on Power Systems, 28, 3 (2013)

15. Wang Jiayu, Gu Xueping, Wang Tao, Zhang Shang Power system critical node identification based on power tracing and link analysis method Power System Protection and Control, 45, 6 (2017)

16. Zou Yanli, Yao Fei, Wang Yang, Wang Ruirui, Wu Lingjie Critical Node Identification for Power Systems Based on Network Structure and Power Tracing Journal of Guangxi Normal University(Natural Science Edition), 37, 1 (2019)

17. Wang, Zhuoyang Chen, Guo Hill, David J. Dong, Zhao Yang A power flow based model for the analysis of vulnerability in power networks Physica, A. Statistical Mechanics \& Its Applications, 460: 105-115 (2016)

18. Li Changchao, Kang Zhongjian, Yu Hongguo, Li Xin, Zhao Bin Identification Method of Key Nodes in Power System Based on Improved PageRank Algorithm TRANSACTIONS OF CHINA ELECTROTECHNICAL SOCIETY, 34, 9 (2019)

19. Lin Hongji, Zhao Yuxuan, Lin Guanqiang, Li Huisong, Mo Tianwen, Ye Xiaojun, Lin Zhenzhi Critical Node Identification in Power Systems Based on Improved Node Importance Contribution Matrix Electric Power Construction, 38, 10 (2017)

20. Li Guiju, Huang Hongguang, Shu Qin. Evaluation method for node importance in power telecommunication network based on interdependent network theory Power System Protection and Control, 47, 11 (2019)

21. Jin Ning, Xiao Xiaoqiang. Evaluation Method of Destruction Resistance of Regional Communication Network Based on Node Shrinkage Method SCIENCE \& TECHNOLOGY INFORMATION, 3 (2019)

22. Wang Guocheng. Research on Power Grid Risk Assessment Considering Cascading Failure. North China Electric Power University(Beijing), (2018)

23. P Bak, C Tang, and K Wiesenfeld, Self-Organized Criticality: an Explanation of the 1/f Noise, Phys Rev Lett, 59: 381-384 (1987)
24. Dorogovtsev, S. N., Goltsev, A. V. \& Mendes, J. F. F. K-core organization of complex networks. Physical Review Letters 96, 040601 (2006)

25. I. Dobson, J. Chen, J. S. Thorp, B. A. Carreras, D. E. Newman Examining criticality of blackouts in power system models with cascading events. Proceedings of the 35th Hawaii International Conference on System Sciences. Maui, Hawaii,: 63-72 (2002)

26. Dobson I, Carreras B A, Newman D E . A probabilistic loading dependent model of cascading failure and possible implications for blackouts. Hawaii International Conference on System Science, Hawaii, 1-8 (2003)

27. Albert R, Kinney R, Crucitti P, Latora V Modeling cascading failures in the North American power grid The European physical journal, B. Condensed matter physics ,46, 1 (2005)

28. Shen Xiaoxiao. Cascading Failures on Complex Networks based on CML Shanghai: Shanghai Jiao Tong University,(2010)

29. Yu, Yi Xiao, Gaoxi Zhou, Jie Wang, Yubo Wang, Zhen System crash as dynamics of complex networks. Proceedings of the National Academy of Sciences 113, 11726-11731 (2016)

30. Zhou Dong, Elmokashfi Ahmed. Network recovery based on system crash early warning in a cascading failure model Scientific reports, 8, 1 (2018) 